\title{
Ocorrência de doenças cardiometabólicas em idosos atendidos em um centro de referência em Belém - PA
}

Occurrence of cardiometabolic diseases in elderly attended at a reference center in Belém - PA

Presencia de enfermedades cardiometabólicas en ancianos atendidos en un centro de referencia en

Belém - PA

Tanise Nazaré Maia Costa

ORCID: https://orcid.org/0000-0003-1319-5591

Centro Universitário do Estado do Pará, Brasil

E-mail: tanise.costa@prof.cesupa.br

Nezilour Lobato Rodrigues

ORCID: https://orcid.org/0000-0003-3715-1075 Hospital Universitário João de Barros Barreto, Brasil

E-mail: nezilour@hotmail.com

Amanda Vallinoto Silva de Araújo

ORCID: https://orcid.org/0000-0003-2638-7912

Centro Universitário do Estado do Pará, Brasil

E-mail: amandavsaraujo2@gmail.com

Andressa Nogueira Barbosa

ORCID: https://orcid.org/0000-0002-1261-8030 Hospital Universitário João de Barros Barreto, Brasil

E-mail: nog.andressa@ hotmail.com

Bárbara Gabriel Mafra

ORCID: https://orcid.org/0000-0002-8120-5168

Centro Universitário do Estado do Pará, Brasil

E-mail: barbaragmafra@gmail.com

Isabella Rocha Gonçalves

ORCID: https://orcid.org/0000-0002-4608-5877

Centro Universitário do Estado do Pará, Brasil

E-mail: isarochag12@gmail.com

Mariana do Nascimento Eiró

ORCID: https://orcid.org/0000-0001-6809-5702

Centro Universitário do Estado do Pará, Brasil

E-mail: marianaeiro9@gmail.com

Victória Oliveira da Costa

ORCID: https://orcid.org/0000-0001-8298-8021

Centro Universitário do Estado do Pará, Brasil

E-mail: victoriaodacosta@gmail.com

\section{Resumo}

Este estudo objetiva identificar a ocorrência de Hipertensão Arterial Sistêmica (HAS), Diabetes Mellitus (DM) e Dislipidemia (DLP) em idosos atendidos, relacionando com sexo e idade. Trata-se de um estudo transversal, observacional e estatístico-descritivo, que tem como base a análise de prontuários de 470 idosos, de ambos os sexos, em acompanhamento em um centro de referência ambulatorial de atenção secundária, em Belém - PA. Foram excluídos do estudo pacientes cujos prontuários possuíam dados inconclusivos. Foi aplicado um formulário de coleta, confeccionado pelos próprios autores, com informações acerca do sexo, idade e presença de HAS, DM e DLP. A análise estatística foi realizada no programa Statistical Package for the Social Sciences. Para verificar a associação entre HAS, DM e DLP e as variáveis independentes sexo e idade, foi realizado o teste de qui-quadrado de aderência. Observou-se que dos 470 indivíduos, $76,2 \%$ eram do sexo feminino e $23,8 \%$, do masculino. A idade variou de 60 a 117 anos, com média de 76,65. A predominância de HAS foi de 56,2\%; de DM2, 20,2\%; e, de DLP, 19,78\%. Diante dos resultados obtidos, observa-se que a ocorrência de HAS, DM e DLP é significativa em idosos independente do sexo. Desta forma, relacionando que as doenças cardiovasculares são causa frequente de morte, se torna de extrema importância o incentivo de medidas governamentais de prevenção e controle dessas doenças.

Palavras-chave: Envelhecimento; Doenças metabólicas; Prevalência.

\section{Abstract}

This study aims to identify the predominance of Systemic Arterial Hypertension (HAS), Diabetes Mellitus (DM) and Dyslipidemia (DLP) in elderly patients, relating to gender and senior group. This is a cross-sectional, observational 
and descriptive-statistical study, established on the investigation of medical records of 470 elderly people, of both genders, being monitored at an outpatient secondary care referral center in Belém - PA. Patients whose medical records had inconclusive data were excluded from the study. A collection form was applied, made by the authors themselves, with information about gender, age and presence of HAS, DM and DLP. Statistical analysis was performed using the Statistical Package for the Social Sciences program. To confirm the connection in the middle of HAS, DM and DLP and the independent variables sex and age, the chi-square test of adherence was registered. It was detected that of the 470 individuals, $76.2 \%$ were female and $23.8 \%$ were male. Age ranged from 60 to 117 years, with an average of 76.65. The occurrence of HAS was 56.2\%; of DM2, 20.2\%; and for DLP, 19.78\%. Reached from the results obtained, it is observed that the prevalence of HAS, DM and DLP is significant in the aged, regardless of gender. Thus, considering that cardiovascular diseases are a frequent cause of death, it is extremely important to encourage policies to prevent and control these diseases.

Keywords: Aging; Metabolic diseases; Prevalence.

\section{Resumen}

Este estudio tiene como objetivo identificar la ocurrencia de hipertensión arterial sistémica (HAS), diabetes mellitus (DM) y dislipidemia (DLP) en pacientes de edad avanzada, en relación con el sexo y la edad. Se trata de un estudio transversal, observacional y descriptivo-estadístico, basado en el análisis de historias clínicas de 470 ancianos, de ambos sexos, en seguimiento en un centro de referencia de atención secundaria ambulatoria en Belém - PA. Los pacientes cuyos registros médicos tenían datos no concluyentes fueron excluidos del estudio. Se aplicó un formulario de recogida, elaborado por los propios autores, con información sobre sexo, edad y presencia de HAS, DM y DLP. El análisis estadístico se realizó mediante el paquete estadístico para el programa de Ciencias Sociales. Para verificar la asociación entre HAS, DM y DLP y las variables independientes sexo y edad, se aplicó lo argumento de adherencia chi-cuadrado. Se observó que de los 470 individuos, el 76,2\% eran mujeres y el 23,8\% eran hombres. La edad osciló entre 60 y 117 años, con una media de 76,65. La prevalencia de HAS fue del 56,2\%; de DM2, 20,2\%; y para DLP, 19,78\%. Con base en los resultados obtenidos, se observa que la prevalencia de HAS, DM y DLP es significativa en los ancianos, independientemente del sexo. Así, al relatar que las enfermedades cardiovasculares son una causa frecuente de muerte, es de suma importancia impulsar políticas de prevención y control de estas enfermedades.

Palabras clave: Envejecimiento; Enfermedades metabólicas; Prevalencia.

\section{Introdução}

O envelhecimento populacional, mundialmente, vem crescendo de forma substancial em tempos atuais. Dados estatísticos revelam transparente alteração no perfil etário dos povos, principalmente pela redução da taxa de natalidade (Guerra et al., 2021). Calcula-se que a população com mais de 60 anos de idade que vivem na América Latina, duplicará até 2025 (Ferreira et al., 2018).

Essa constatação pode-se estender até o Brasil, onde atualmente, a expectativa de vida do cidadão do país é de 74,9 anos (Malachias et al., 2016). Este aumento da expectativa não é um fenômeno repentino ou inesperado; pelo contrário, resulta das inúmeras transformações demográficas ocorridas nas décadas pregressas, motivo pelo qual, na maioria dos países, trata-se de um processo inexorável (Chaimowicz et al., 2013).

Exatamente, essa maior expectativa de vida modificou o perfil de morbi-mortalidade, caracterizado por um aumento de doenças crônico-degenerativas em detrimento das infectocontagiosas, tornando-se em prevalentes e importantes problemas de saúde pública em todos os países, independentemente de seu grau de desenvolvimento (Melo et al., 2018; Toscano, 2004; Pimenta et al., 2015). Tal evento gera efeitos diretos na qualidade de vida da população idosa e desafia tanto os sistemas de saúde quanto de previdência social (Miranda, Mendes \& Silva, 2016).

Concordante a isso, em 2014, cerca de 79,8\% das mortes no Brasil foram atribuídas às Doenças Crônicas Não Transmissíveis (DCNT), entre elas, as doenças cardiovasculares (DCV) são as principais causas de morbi-mortalidade no Brasil. Sendo a hipertensão (HAS), a diabetes mellitus (DM), a dislipidemia (DLP) e o tabagismo os fatores de risco cardiovasculares de maior importância (Ferreira et al., 2018; Martinez \& Murad, 2014; Melo et al., 2019).

As DCNT, no entanto, compartilham não apenas os diversos fatores de risco, como hereditariedade, raça, sexo, consumo excessivo de álcool, sedentarismo, entre outros, mas também a fisiologia (Martinez \& Murad, 2014; Melo et al., 
2019). Ao avaliar a occorrência de HAS, DM e DLP, por exemplo, como um grupo de patologias, pode haver interseção entre elas, possivelmente por terem mecanismos fisiopatológicos subjacentes (Martinez \& Murad, 2014).

Como exemplo, é conhecido que a pressão arterial é determinada pelo produto da resistência vascular periférica (RVP) e do débito cardíaco (Sanjuliani, 2002). A RVP, por sua vez, pode ser modificada em condições de alteração na estrutura dos vasos (remodelamento, hipertrofia), fato que é influenciado na presença de hiperinsulinemia, evidenciando a relação entre HAS e DM (Martinez \& Murad, 2014).

Já em relação a DLP, a sua fisiopatologia consiste, sobretudo, em uma ação predominante no endotélio, nas células espumosas e, por fim, na formação da placa ateroscletórica. Ao passo que HAS e DM também atuam no endotélio promovendo a disfunção do mesmo, por intermédio de processos inflamatórios e estresse oxidativo, torna-se clara a conexão entre as fisiopatologias referidas (Martinez \& Murad, 2014).

A respeito do DM, sabe-se que é um distúrbio que atinge muitas pessoas no mundo, sendo caracterizada por elevação da glicemia, resultante da deficiência hormonal ou em alteração na utilização da insulina. O DM tipo 2 (DM2) é marcada por um quadro de resistência insulínica, o qual acomete, principalmente adultos, idosos e pessoas com risco de obesidade ou obesas, correspondendo a quase $85 \%$ a $90 \%$ dos casos, constituindo-se hoje um grave problema de saúde pública (Jacob, Breuer, \& Kostev, 2016).

Diante disso, demonstra-se que a presença das doenças crônico-degenerativas no idoso é um assunto de destaque, o qual necessita de uma atenção conjunta para a redução dos fatores de risco e para a prevenção de suas complicações, sendo a promoção conjunta do tratamento da HAS, DM e DLP um importante pilar para a diminuição da morbimortalidade (Melo et al., 2019). Portanto, conhecer as condições de disposição física e mental da população idosa é essencial para aprimorar a qualidade de vida, diminuir a mortalidade e guiar políticas públicas de prevenção e controle da hipertensão.

Nesse contexto, este estudo objetiva identificar a prevalência de HAS, DM2 e DLP em idosos atendidos em um centro de referência ambulatorial, no município de Belém - PA, relacionando com sexo e idade.

\section{Metodologia}

Trata-se de um estudo transversal, observacional e estatístico-descritivo, que como ponto de partida de um universo de 950 pessoas idosas cadastradas, tem como base a análise de prontuários de 470 idosos. Foram incluídos os indivíduos com idade maior ou igual a 60 anos, de ambos os sexos, em acompanhamento em um centro de referência ambulatorial de atenção secundária, na cidade de Belém - PA, e que aceitaram participar do estudo. Foram excluídos do estudo pacientes cujos prontuários possuíam dados inconclusivos.

Os dados foram coletados após aprovado pelo Comitê de Ética em Pesquisa do Centro Universitário do Estado do Pará (CAAE - 15933219.6.0000.5169), através de um formulário, confeccionado pelos próprios autores, contendo informações acerca do sexo, idade e presença de Hipertensão Arterial Sistêmica, Diabetes Mellitus e Dislipidemia. A presença de tais patologias foi considerada mediante diagnóstico prévio registrado no prontuário.

Os dados foram tratados por meio do programa Microsoft Office Excel 2017. A análise estatística foi realizada no programa SPSS (Statistical Package for the Social Sciences). Para verificar a existência de associação entre Hipertensão, Diebetes e Dislipidemia e as variáveis independentes sexo e idade, foi aplicado o teste de qui-quadrado de aderência. A significância estatística foi aceita ao nível de 95\% (p-valor < 0,05). 


\section{Resultados}

Dos 470 indivíduos elegíveis para o estudo, 358 (76,2\%) eram do sexo feminino e 112 (23,8\%), do sexo masculino. A idade variou de 60 a 117 anos, com média de 76,65 (DP=7,87). Quanto à faixa etária, 102 idosos apresentavam idade entre 60 e 69 anos e 368 idosos apresentavam 70 anos ou mais.

A predominância de HAS foi de 56,2\% (n 264); de DM2, 20,2\% (n 95); e, de DLP, 19,78\% (n 93) como demonstra a Figura 1. Ao analisar a prevalência simultânea de Hipertensão Arterial Sistêmica, Diabetes Mellitus e Dislipidemia, 10\% dos idosos apresentavam o diagnóstico de DM e HAS, 1,7\% apresentavam DM e Dislipidemia, 7,4\% idosos apresentavam HAS e Dislipidemia e 3,8\% idosos obtinham os três diagnósticos.

Figura 1: Prevalência de Hipertensão arterial sistêmica, diabetes mellitus tipo 2 e dislipidemia em idosos.

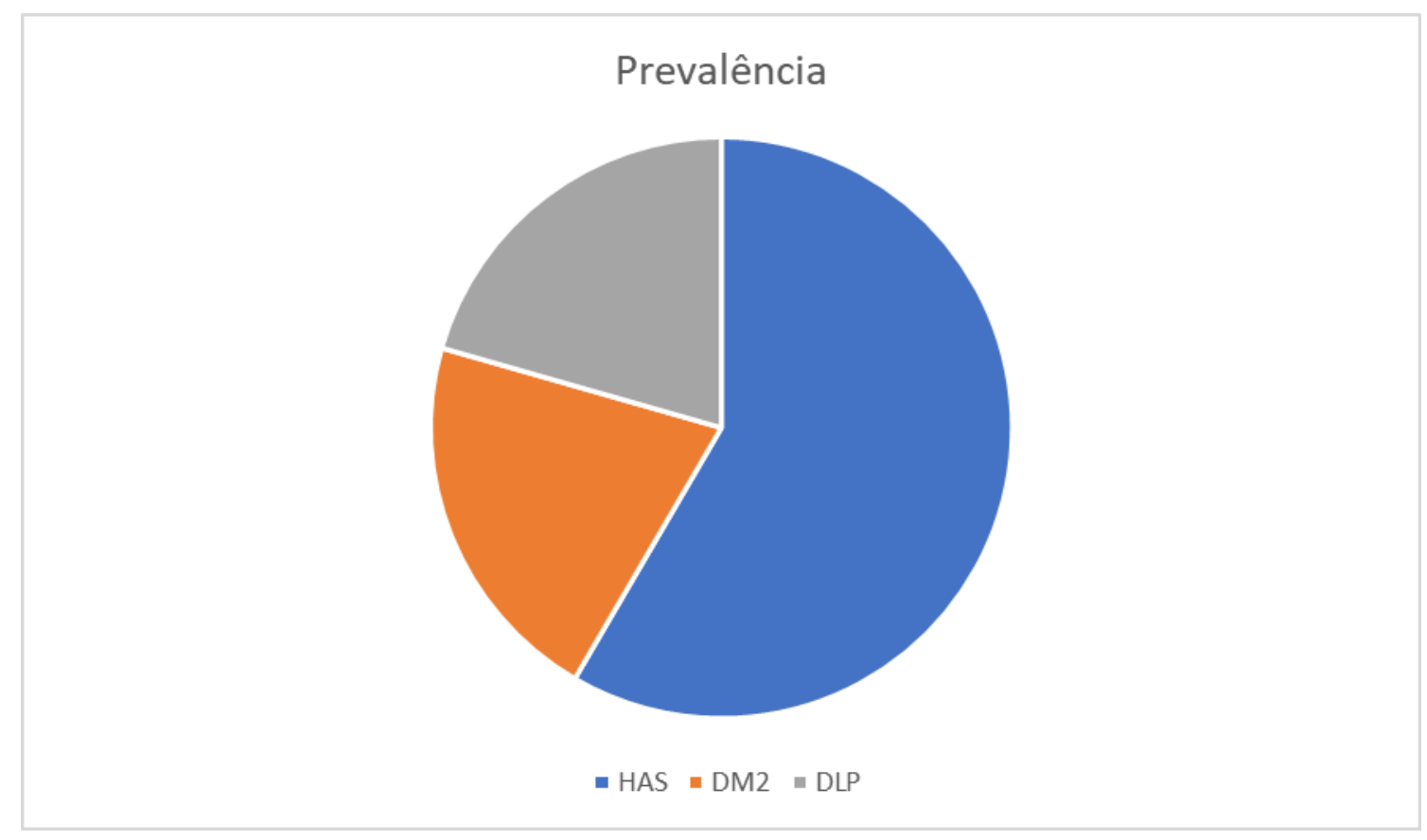

Fonte: Protocolo de pesquisa.

A ocorrência de HAS de acordo com sexo, foi de 56,1\% para o feminino e 56,3\% para o masculino. Não houve diferença estatisticamente significativa entre os sexos $(\mathrm{p}=0,98)$. Ao comparar faixas etárias, foi identificada uma prevalência semelhante $(p=0,77)$ em idosos entre 60 e 69 anos e aqueles com idade igual ou superior a 70 anos (Tabela 1).

Tabela 1: Prevalência de Hipertensão arterial sistêmica por sexo e faixa etária.

\begin{tabular}{|c|c|c|c|c|}
\hline \multirow{2}{*}{ Variável } & $\mathbf{n}$ & \multicolumn{2}{|c|}{ Hipertensão Arterial Sistêmica } & \multirow{2}{*}{ p-valor } \\
\hline Sexo feminino & 358 & 201 & $56,1 \%$ & \multirow{2}{*}{0,98} \\
\hline Sexo masculino & 112 & 63 & $56,3 \%$ & \\
\hline 60-69 anos & 102 & 56 & $54,9 \%$ & \multirow{2}{*}{0,77} \\
\hline$\geq 70$ anos & 368 & 208 & $56,5 \%$ & \\
\hline
\end{tabular}

Fonte: Protocolo de pesquisa.

Acerca da DM, a prevalência encontrada foi de $20,2 \%$, sendo $20,1 \%$ do sexo feminino e $22,6 \%$ do sexo masculino (p= 0,92). Quanto às faixas etárias, $23,5 \%$ dos idosos abaixo de 70 anos apresentavam Diabetes, enquanto 19,2\% dos idosos com 
idade igual ou superior a 70 anos obtiveram esse diagnóstico $(\mathrm{p}=0,34)$ (Tabela 2).

Tabela 2: Prevalência de Diabetes mellitus tipo 2 por sexo e faixa etária.

\begin{tabular}{|c|c|c|c|c|}
\hline Variável & $\mathbf{n}$ & \multicolumn{2}{|c|}{ Diabetes Mellitus } & \multirow{2}{*}{ p-valor } \\
\hline Sexo feminino & 358 & 72 & $20,1 \%$ & \multirow{2}{*}{0,92} \\
\hline Sexo masculino & 112 & 23 & $22,6 \%$ & \\
\hline 60-69 anos & 102 & 24 & $23,5 \%$ & \multirow{2}{*}{0,34} \\
\hline$\geq 70$ anos & 368 & 71 & $19,2 \%$ & \\
\hline
\end{tabular}

Fonte: Protocolo de pesquisa.

No que tange a Dislipidemia, 20,1\% das mulheres apresentavam esse diagnóstico, enquanto 20,5\% dos homens foram acometidos $(\mathrm{p}=0,92)$. Na comparação entre as faixas etárias, também não foi observada diferença significativa $(\mathrm{p}=0,53)$

(Tabela 3).

Tabela 3: Prevalência de Dislipidemia por sexo e faixa etária.

\begin{tabular}{|c|c|c|c|c|}
\hline Variável & $\mathbf{n}$ & \multicolumn{2}{|c|}{ Dislipidemia } & \multirow{2}{*}{ p-valor } \\
\hline Sexo feminino & 358 & 72 & $20,1 \%$ & \multirow{2}{*}{0,75} \\
\hline Sexo masculino & 112 & 21 & $18,8 \%$ & 0,75 \\
\hline 60-69 anos & 102 & 18 & $17,6 \%$ & \multirow{2}{*}{0,53} \\
\hline$\geq 70$ anos & 368 & 75 & $20,3 \%$ & \\
\hline
\end{tabular}

Fonte: Protocolo de pesquisa.

\section{Discussão}

O Brasil, conforme o Ministério da Saúde, está perante uma epidemia de DCNT. Devido a isso, há um planejamento de combate a essa realidade através do Plano de Ações Estratégicas para o Enfrentamento das Doenças Crônicas em 2011. Essa constatação alarmante ocorre por aumento vertiginoso, de 2000 a 2010, das prevalências de doenças cardiometabólicas, provavelmente corroboradas pela melhoria no diagnóstico precoce (Guerra et al., 2021). Essas informações concordam com as prevalências tanto isoladas quanto em conjunto, da investigação realizada pela atual pesquisa.

Justamente acerca da prevalência simultânea das patologias, a saber: HAS, DM e DLP, o trabalho apresentou quase 4\% dos idosos entrevistados com tais doenças e, de forma global, tais patologias aumentam a chance desses pacientes desenvolverem complicações cardiovasculares se não for feito acompanhamento adequado de cada DCNT. Se compararmos com o estudo feito na Bahia com 252 idosos, também houve uma parcela de idosos que apresentam tais doenças e o autor do estudo também destaca a ameaça de agravamento para tais idosos, tal preocupação é decorrente principalmente da obesidade visceral, sendo esta variante um fator de agravo que se correlaciona com múltiplos outros fatores de risco cardiovascular. Logo, a obesidade visceral está intimamente relacionada com as três principais DCNTs listadas aqui no presente estudo (Silva et al., 2020).

Ainda no presente estudo, quanto ao quadro de HAS, não foi evidenciada diferença na prevalência entre os sexos, sendo verificado também em um estudo com 354 idosos no Vietnã e em outro estudo realizado no município de São Paulo com 121 idosos (Bui Van et al., 2019; Santos et al., 2020). Entretanto, diverge de estudo realizado em Goiânia com 912 idosos, no qual a prevalência em homens $(78,6 \%)$ foi maior do que nas mulheres $(72,6 \%)(p=0,043)$ (Sousa et al., 2018). Além do mais, foi observado no atual estudo que a HAS é a doença cardiovascular mais prevalente entre a população idosa, sendo observado 
também em um estudo com 20 idosos de uma instituição de longa permanência no município de João Pessoa, ao qual 75\% dos idosos apresentaram o diagnóstico de HAS (Viana, Figueiredo, \& Ferreira, 2017). Relacionado a tal prevalência da HAS em idosos, pode-se associar a fatores de risco como: sedentarismo, avanço da idade e má alimentação que contribuem para o aparecimento de tal doença.

Quanto às faixas etárias, no estudo realizado no Vietnã, foi verificado um aumento proporcional considerável de HAS com a idade, o que não foi evidenciado no nosso estudo.

Quanto a prevalência de Diabetes, não houve diferença significativa na comparação entre as faixas etárias, como foi identificado também em um trabalho realizado na China, no qual foi observado prevalência de 23,7\% no total (Xiaolei et al., 2016). No estudo presente, não foi encontrado também diferença entre os sexos, o que se contrapõe a um estudo na capital do Rio Grande do Sul, com 763 idosos, no qual a prevalência foi maior em mulheres $(27,2 \%)$ do que nos homens $(17 \%)(\mathrm{p}=$ 0,001) (Silva et al., 2016).

Ademais, acerca do DM, foi observado também no presente estudo que a patologia estava presente em torno de 20,2\% dos idosos, sendo a segunda doença mais prevalente entre as DCNTs. Tal dado também foi identificado em estudo, em que foram entrevistados 254 idosos de dois municípios do estado da Bahia, revelando que 15,8\% deles apresentaram a doença (Silva et al., 2020).

A diabetes destaca-se como uma pandemia, a OMS estima que 346 milhões de pessoas possuam a doença. A prevalência dos casos do diabetes está relacionada a uma gama de fatores, sejam eles de âmbito individual ou de forma global, e tais fatores estão presentes na sociedade moderna, como por exemplo: o envelhecimento populacional, o crescimento acelerado da urbanização, dietas hipercalóricas com baixo poder nutritivo, presença de sedentarismo e obesidade e altos índices de fatores estressantes durante o dia.

Quanto ao cenário relacionado a dislipidemia, também não houve diferença significativa entre os sexos. Em comparação com outros trabalhos, a prevalência foi menor. Em um realizado na Alemanha com idosos maiores de 65 anos, a prevalência foi de aproximadamente $40 \%$ em ambos os sexos (Jacob, Breuer, \& Kostev, 2016). Quanto ao índice de pacientes que apresentam em conjunto dislipidemia e diabetes, foi visto que tal concomitância é a de menor prevalência no presente estudo, sendo contraposta a um estudo publicado pela Revista Saúde Integrada, ao qual avaliaram 43 pacientes do município de Santo Ângelo, no estado do Rio Grande do Sul, e apresentaram que a maioria dos participantes do estudo tinham as duas doenças (Valença et al., 2018). Tal relação pode ser explicada devido ao fato de que os pacientes do nosso estudo não apresentaram também, além da DM e DLP, a obesidade, variável esta que está presente no estudo na cidade de Santo Ângelo e que é um fator de risco para o desenvolvimento da dislipidemia, visto que em pacientes diabéticos há mais chance de se ter anormalidades no metabolismo dos lipídios, pois a resistência insulínica e o déficit de tal hormônio associado com a obesidade, estão relacionados a um perfil característico com padrão de hiperlipidemia mista, HDL-c baixo e ocasionalmente com níveis elevados de LDL (Valença et al., 2018).

\section{Conclusão}

Diante dos resultados obtidos, observa-se que a prevalência de HAS, Diabetes e Dislipidemia é significativa na faixa etária idosa independente do sexo. A partir desse contexto, relacionando ao fato de que as doenças cardiovasculares são causa frequente de morte, se torna de extrema importância o incentivo de medidas governamentais de prevenção e controle dessas doenças.

A qualidade de vida do idoso está intrinsicamente relacionada com a presença dessas doenças, pois o declínio das funções cognitivas pode ser afetado pela existência de doenças crônicas. A partir disso, a redução dos fatores de risco para 
essas patologias é essencial, com o intuito de reduzir esses números pela mudança de hábito da população e a maior conscientização sobre o cuidado com a própria saúde desde as faixas etárias mais novas.

Além do mais, faz-se necessário mais estudos científicos que possam discutir no âmbito acadêmico, a prevenção de complicações cardiovasculares decorrentes de distúrbios cardiometabólicos na população idosa, principalmente na atenção primária de saúde, incentivando maior atenção e cuidado pelos serviços de saúde, serviços sociais, a fim de possibilitar um processo de envelhecimento mais saudável e com maior autonomia e independência dos idosos.

\section{Referências}

Bui Van, N.. et al. (2019). Prevalence and Risk Factors of Hypertension in the Vietnamese Elderly. High Blood Pressure \& Cardiovascular Prevention: The Official Journal of the Italian Society of Hypertension. 26(3), 239-46. 10.1007/s40292-019-00314-8.

Chaimowicz, F., Barcelos, E. M., Madureira, M. D. S., \& Ribeiro, M. T. F. (2013). Saúde do Idoso. (2ª ed.): UFMG.

Ferreira, S. R. G., Chiavegatto Filho, A. P. D., Lebrão, M. L., Duarte, Y. A. O., \& Laurenti, R. (2018). Doenças cardiometabólicas. Rev Bras Epidemiol, 21(Suppl 2), E180008. 10.1590/1980-549720180008.supl.2

Guerra., et al. (2021). Envelhecimento: interrelação do idoso com a família e a sociedade. Research, Society and Development, 10(1), e3410111534. http://dx.doi.org/10.33448/rsd-v10i1.11534

Jacob, L., Breuer, J., \& Kostev, K. (2016). Prevalence de doenças crônicas entre pacientes mais velhos em práticas gerais alemãs. Ger Med Sci. 14. https://doi.org/10.3205/000230.

Malachias, M. V. B., Souza, W. K. S. B., Plavnik, F. L., Rodrigues, C. I. S., et al. (2016). $7^{\text {a }}$ Diretriz Brasileira de Hipertensão Arterial. Arq Bras Cardiol.

Martinez, L. R. C., \& Murad, N. (2014). Hipertensão, diabetes e dislipidemia técnico envolvido. Rev bras hipertens. $92-7$.

Melo, E. M. de A., Marques, A. P. de O., Leal, M. C. C., \& Melo, H. M. de A. (2018). Síndrome da fragilidade e fatores associados em idosos residentes em instituições de longa permanência. Saúde Debate. 42, 468-80. https://doi.org/10.1590/0103-1104201811710.

Melo, S. P. da S. de C., Cesse, E. Â. P., Lira, P. I. C., Rissin, A., Cruz, R. de S. B. L. C., \& Batista Filho, M. (2019). Doenças crônicas não transmissíveis e fatores associados em adultos numa área urbana de pobreza do nordeste brasileiro. Ciênc Saúde Coletiva. 24, 3159-68. https://doi.org/10.1590/141381232018248.30742017 .

Miranda, G. M. D., Mendes, A. da C. G., \& Silva, A. L. A. da. (2016). Envelhecimento populacional no Brasil: desafios sociais atuais e futuros e consequências. Rev Bras Geriatr Gerontol. 19, 507-19. https://doi.org/10.1590/1809-98232016019.150140

Pimenta, F. B., Pinho, L., Silveira, M. F., \& Botelho, A. C. de C. (2015). Fatores associados a doenças crônicas em idosos atendidos pela Estratégia de Saúde da Família. Ciênc Saúde Coletiva. 20, 2489-98. https://doi.org/10.1590/1413-81232015208.11742014.

Sanjuliani, A. F. (2002). Fisiopatologia da hipertensão arterial: conceitos teóricos úteis para a prática clínica. Rev SOCERJ. 210-8.

Santos, A. N. M., Nogueira, D. R. C., Gutierrez, B. A. O., Chubaci, R. Y. S., \& Oliveira, C. R. B. (2020). Cardiometabolic diseases and active aging polypharmacy in control. Rev Bras Enferm. 73(2), e20180324. http://dx.doi.org/10.1590/0034-7167-2018-0324

Silva, A. B. da., Engroff, P., Sgnaolin, V., Ely, L. S., Gomes, I., \& Silva, A. B. da., et al. (2016). Prevalência de diabetes mellitus e adesão medicamentosa em idosos da Estratégia Saúde da Família de Porto Alegre / RS. Cadernos Saúde Coletiva. 24, 308-16. https://doi.org/10.1590/1414-462x201600030017.

Silva, D. dos S., et al. (2020). Alterações metabólicas e cardiovasculares e sua relação com a obesidade em idosos. Brazilian Journal of Health Review. 3(3), 4357-69.

Sousa, A. L. L., Batista, S. R., Sousa, A. C., Pacheco, J. A. S., Vitorino, P. V. de O., \& Pagotto, V. (2018). Prevalência, tratamento e controle da hipertensão em idosos em uma capital brasileira. Arquivos Brasileiros de Cardiologia. https://doi.org/10.5935/abc.20180274

Toscano, C. M. (2004). As campanhas nacionais para detecção das doenças crônicas não-transmissíveis: diabetes e hipertensão arterial. Ciênc Saúde Coletiva. 9, 885-95. https://doi.org/10.1590/S1413-81232004000400010.

Valença, T. V. R., et al. (2018). Obesidade, diabetes e hipertensão associados a dislipidemia e dano hepático. Revista saúde integrada. 11(22), 2-18. http://local.cnecsan.edu.br/revista/index.php/saude/index

Viana, M. M. L., Figueiredo, M. S. B. R., \& Ferreira, D. (2017). Parâmetros cardiovasculares em idosos institucionalizados: identificar, monitorar e prevenir. Anais Cong Int Humano. https://www.editorarealize.com.br/editora/anais/cieh/2017/TRABALHO_EV075_MD2_SA4_ID2359_03102017141919.pdf

Xiaolei, X., et al. (2016). Investigation and Analysis on the Prevalence of Diabetes among the Elderly in Chengyang District of Qingdao City. Journal of Nursing. 5(1), nursing.usp-pl.com, 10.18686/jn.v5i1.84. 